\title{
Cancer nanomedicine: Is targeting our target?
}

\author{
Twan Lammers ${ }^{1,2,3,{ }^{*}, \text { Fabian Kiessling }^{1} \text {, Marianne Ashford }}{ }^{4}$, Wim Hennink ${ }^{3}$, Daan \\ Crommelin $^{3,}{ }^{*}$, and Gert Storm ${ }^{2,3}$
}

1Department of Nanomedicine and Theranostics, Institute for Experimental Molecular Imaging, RWTH Aachen University Clinic, Aachen, Germany ${ }^{2}$ Department of Targeted Therapeutics, MIRA Institute for Biomedical Technology and Technical Medicine, University of Twente, Enschede, The Netherlands ${ }^{3}$ Department of Pharmaceutics, Utrecht Institute for Pharmaceutical Sciences, Utrecht University, Utrecht, The Netherlands ${ }^{4}$ Pharmaceutical Science, Innovative Medicines, AstraZeneca, Macclesfield, SK10 2NA, UK

\section{Abstract}

Nanomedicine may have a delivery problem. Rigorous, realistic and holistic rethinking is needed to improve nanomedicine performance and increase patient benefit.

\section{Introduction}

A major driving force for research into nanomaterials has been their potential use as drug delivery vehicles, in particular for drug targeting to tumours. The efficacy of such targeted approaches in cancer therapy and, more specifically, in solid tumour targeting, has become a discussion point in the field1. Despite significant financial investment, only a few of the tumour-targeted nanomedicine formulations developed have become drug products2. Moreover, a recently published controversial meta-analysis shows that in preclinical models, on average, only $0.7 \%$ of the injected dose (ID) of intravenously administered nanoparticles accumulates in tumours3.

Is this seemingly low number the reason why tumour-targeted nanomedicines have not (yet) been very successful in the clinic? We would argue, probably not. Antibodies, which are in a way also nanomedicines, have had considerable clinical and commercial success (annual revenues of around US\$20 billion for solid tumour therapy alone) but do not target tumours more efficiently. Both in mice and men, tumour concentrations generally range between 0.07 and 7\%ID - similar to clinically used liposome formulations as well as other established nanomedicines. We should therefore be careful not to let ourselves be misled by numbers, because numbers as such do not matter. In this context, one could ask the question whether the preclinical picture or the current clinical situation would be any different if we could have achieved a tumour accumulation of 7\%ID instead of the now reported $0.7 \%$ ID

\footnotetext{
*Corresponding authors: tlammers@ukaachen.de, d.j.a.crommelin@uu.nl.

Competing interests statement:

The authors declare no competing interests.
} 
What counts is patient benefit. This is largely independent of the percentage of the injected dose that can be delivered to tumours, but instead results from a shift in the balance between off- and on-target accumulation. Nanomedicines, like antibodies, are able to increase drug concentrations at pathological sites, from values (well) below 0.1\% ID for intravenously administered small molecule drugs, to values exceeding 1\%ID for targeted drugs. If such increases in target site accumulation are paralleled by a reduced localization in potentially endangered healthy tissues, increases in patient benefit can be achieved. If nanomedicines can also be used to deliver highly potent 'non-classical' drugs to tumours, if they are rationally and more extensively incorporated in combination regimens and/or if they are only given to preselected patients that are (more) likely to respond to tumour-targeted treatments, patient benefit can be further enhanced.

\section{Past progress}

By shifting the balance between off- and on-target localization, several nanomedicines have become reasonably successful in the clinic. For example, PEGylated liposomal doxorubicin (Doxil) attenuates drug accumulation in the heart, while attaining or improving tumour concentrations. In contrast to free doxorubicin, for which cardiomyopathy is the main doselimiting side effect, the liposomal form is better tolerated and enables prolonged drug dosing, together rendering it more suitable for integration in combination therapies. Individuals treated with Doxil also lose less hair, which is often considered to be of subordinate importance, but does improve the quality of life of a patient.

Similarly, Abraxane has become very useful for increasing patient benefit. Abraxane is a 130-nm-sized co-condensate of albumin and paclitaxel, which is fairly unstable in blood and possesses similar pharmacokinetic properties to Taxol (that is, the clinically routinely used Cremophor EL-based formulation of paclitaxel); therefore, it does not result in more efficient solid tumour targeting. However, in terms of patient benefit, Abraxane has several clear advantages over Taxol. These include a shorter infusion time, less need for corticosteroid and/or antihistaminic comedication, the ability to increase drug doses and a more favourable performance in combination regimens. Consequently, despite being a nanomedicine formulation that may not always ensure a tumour accumulation exceeding $0.7 \% \mathrm{ID}$, Abraxane has become a clinical and commercial success, with annual revenues in the order of US\$1 billion.

\section{Current concepts}

The majority of nanomedicines in (pre)clinical trials are based on established drugs, such as doxorubicin and paclitaxel. Does that make sense? To some extent yes, because a lot of information is available on their pharmacokinetics (PK), pharmacodynamics (PD), side effects and clinical performance. However, we should also think beyond established drug molecules, and look for agents that are highly potent, but fall short in vivo because of undesired PK properties and/or unacceptable efficacy-to-toxicity profiles. Such 'carrierdependent' drugs include nucleotide-based bioactives and highly potent but too toxic chemotherapeutic drugs, such as the tubulin-binding antimitotics mertansine and monomethyl auristatin E. If conjugated to antibodies, tumour concentrations from 0.07 to 
7\%ID can be achieved, and drug accumulation in critical healthy organs can be avoided, resulting in nanoformulations that are more efficient and better tolerated. Consequently, the antibody-drug conjugates Kadcyla (trastuzumab emtansine) and Adcetris (brentuximab vedotin) have become very successful for treating severely ill patients and have improved patient benefit.

Most cancers are treated with combination therapies. Nanomedicines - as a result of their ability to change the spectrum of side effects - may be useful in combination with other treatment modalities. Indeed, both Doxil and Abraxane are routinely used and have performed very well in the clinic in combination regimens. Other nanomedical drug products, such as Onivyde (liposomal irinotecan) have also shown good performance in combination therapies, with improved response rates and clearly increased patient benefit. Furthermore, second-generation nanomedicines specifically designed for combination therapy have done very well: Vyxeos liposomes, for example, which are co-loaded with a synergistic 5:1 molar ratio of cytarabine and daunorubicin, significantly increased survival times in high-risk acute myeloid leukaemia patients in a recently completed phase III trial. These insights, together with ongoing efforts to combine nanoformulations with radiotherapy, hyperthermia and/or immunotherapy, suggest a bright future for nanomedicine in combined-modality anticancer therapy.

\section{Future directions}

Patient selection is the key to improving the performance of nanomedicine in the clinic4,5. This concept is routinely used in the field of molecularly targeted therapeutics. For example, trastuzumab (Herceptin) only works well if patients are selected via immunohistochemical staining of the human epidermal growth factor receptor (HER2, also known as ERBB2) in biopsies. Without biopsy-based preselection, only 10-15\% of patients with breast cancer derive benefit from Herceptin treatment, no more than other therapies. By contrast, with patient selection, more than $50 \%$ of patients respond to treatment. Such companion diagnostics have been established for many other molecularly targeted therapeutics and are also urgently needed to improve the performance of nanomedicine formulations. In this context, biopsy-based information (for example, on vascular, macrophage and/or collagen density) may be helpful, but no systematic studies correlating such biomarkers with nanomedicine accumulation and efficacy are available.

Alternatively, by using imaging agents as companion diagnostics (for example, nanometresized probes for computed tomography, magnetic resonance imaging and positron emission tomography), patients that are likely to respond to nanomedicine therapies can be preselected4,5. Moreover, patients that are unlikely to respond because of low or no target site localization can be excluded; this may be particularly useful for promoting progression through clinical trials by ensuring that only individuals with sufficiently high levels of accumulation in tumours and metastases are included. Accumulation in metastases is especially important: patients in clinical trials often suffer from metastatic disease, and metastasis is the most lethal aspect of cancer. Imaging is the only tool we have at hand to find metastases; without it, it is impossible to evaluate if antimetastatic therapies work. 
Imaging is consequently considered to be of key importance for bridging the gap between nanomedicine and more effective cancer therapy.

To enable patients to benefit from nanomedicines, we have to make clinical translation more efficient, without over-interpreting tumour accumulation and therapeutic efficacy results obtained in (poorly predictive) animal models. We also need to keep issues related to production protocol, patient selection and combination therapy firmly in mind, from the beginning of the translational drug development process onwards. This may require a somewhat different mindset, moving away from focusing on cutting-edge innovation at the academic and preclinical levels, towards more dedicated and systematic efforts to really reach the patient.

\section{Conclusions}

Patients do not benefit from targeting as such, and a reported tumour accumulation of $0.7 \%$ ID does not mean that nanomedicines do not work. We have to think beyond targeting, and beyond numbers, and focus on carrier-dependent drugs, combination therapies, protocols for patient selection and ways to enable rapid and more efficient clinical translation. The now widely accepted 'open innovation model' used in research and development departments in the pharmaceutical industry, and the evolving concept of public- private partnerships, may be instrumental in reaching our overall goal: to make nanomedicines that make a difference for patients.

\section{Acknowledgements}

TL gratefully acknowledges support by the European Research Council (StG-309495, PoC-680882) and by the German Research Foundation (LA 2937/1-2).

\section{References}

1. Torrice M. Does nanomedicine have a delivery problem? ACS Cent Sci. 2016; 2:434-437. [PubMed: 27504489]

2. Venditto VJ, Szoka FC. Cancer nanomedicines: so many papers and so few drugs! Adv Drug Deliv Rev. 2013; 65:80-88. [PubMed: 23036224]

3. Wilhelm S, et al. Analysis of nanoparticle delivery to tumours. Nat Rev Mater. 2016; 1:16014.

4. Lammers T, Rizzo LY, Storm G, Kiessling F. Personalized nanomedicine. Clin Cancer Res. 2012; 18:4889-4894. [PubMed: 22829203]

5. Tietjen GT, Saltzman WM. Nanomedicine gets personal. Sci Transl Med. 2015; 7:314fs47. 